\title{
RESVERATROL AND LORATADINE EFFECTS ON OXIDATIVE STRESS INDUCED BY EXPERIMENTAL INFLAMMATION
}

\author{
DANIELA-RODICA MITREA ${ }^{\mathrm{a}, *}$, SIMONA CLICHICl ${ }^{a}$, \\ ADRIANA FILIPa, DIANA OLTEANU ${ }^{a}$, IOANA BÂLDEA ${ }^{a}$, \\ REMUS MOLDOVAN ${ }^{\mathrm{a}}$, NICOLETA DECEA ${ }^{\mathrm{a}}$, OANA-ALINA HOTEIUC ${ }^{a}$
}

\begin{abstract}
Resveratrol is a polyphenol with important antioxidant and antiinflammatory roles. Loratadine, as a selective inverse-agonist of peripheral receptor $\mathrm{H}_{1}$, is described as an anti-allergic substance with potential antiinflammatory effects. We aimed to assess the protective capacity of loratadine against oxidative stress produced by inflammation with carrageenan in comparison with a well-known antioxidant compound, resveratrol.

Our results showed that resveratrol and loratadine had no protective anti-inflammatory effects in inflamed skin. As expected, resveratrol had antioxidant effects in serum and in liver. Loratadine developed oxidative stress in tegument and serum.

In this experimental model, our results do not sustain the initial hypothesis that loratadine could have a protective effect in inflammation, and, in the contrary, is capable of increasing it, by producing an oxidative stress.
\end{abstract}

Keywords: antioxidants, inflammation, loratadine, oxidative stress, resveratrol

\section{INTRODUCTION}

Inflammation is a common process characterized by vasodilation, leukocytes infiltration and cytokines release and, also by reactive oxygen species production that in turn can initiate or maintain the inflammatory process. Histamine is one of the chemical mediators that are immediately

\footnotetext{
a Iuliu Haţieganu University of Medicine and Pharmacy, Department of Physiology, 1 Clinicilor Street, 400006 Cluj-Napoca, Romania

"Corresponding author: rdmitrea@yahoo.co.uk
} 
released after basophils and mast cells activation that occurs during inflammation. Histamine interacts with histamine receptors that are part of GPCRs (G-protein coupled receptors) that will stimulate the $\mathrm{Ca}^{++}$release from endoplasmic reticulum. The intracellular increase of $\mathrm{Ca}^{++}$concentration allows the amplification of inflammation [1]. Resveratrol (trans-3,4',5-trihydroxystilbene) is a natural polyphenol with antioxidant and some anti-inflammatory effects. Resveratrol has anti-inflammatory effects due to its capacity of inhibiting the pro-inflammatory mediators and the enzymes responsible for the synthesis of these mediators. Resveratrol inhibits transcription factor NF-kB (Nuclear Factor kappa-light chain enhancer of activated B cells), a protein complex involved in cellular response to noxious stimuli that regulates genes responsible for immunity and oxidative stress [2] [3]. Resveratrol also inhibits some activated immune cells [4]. Loratadine (selective inverse-agonist of peripheral receptor $\mathrm{H}_{1}$ ) is used in allergy treatment. Desloratadine, the active metabolite of loratadine, also inhibits the basal and histamine-increased activity of NF-kB [5].

Our study used resveratrol and loratadine to test their efficiency in neutralization of the oxidative stress generated by inflammation, induced by carrageenan injections. The study has on its basis the idea that resveratrol has an important antioxidant role and anti-inflammatory effects. Loratadine was described in literature as anti-inflammatory agent in several allergic diseases and based on these data, the study aim to explore the antioxidant and antiinflammatory effect of loratadine on a model of experimentally induced inflammation, in comparison with the well-studied effect of resveratrol.

In our study, oxidative stress was evaluated through the measurement of the parameters of pro-oxidant and anti-oxidant statuses and a paw inflammation was produced to assess if the pre-treatment using these compounds can be useful in the reducing of the development of inflammatory lesions.

\section{RESULTS AND DISCUSSION}

\section{Plethysmometry}

The measurements made at two hours after inflammation of the posterior right paw, presented a significant increase of the paw volume in rats that received resveratrol and in the group that received loratadine, in comparison with the Control group that suffered only inflammation without treatment. There were not recorded significant differences between the rats treated with resveratrol or with loratadine. 
The plethysmometric values at 24 hours after inflammation showed minor modifications among the groups, without significant differences (Figure 1).

PLETHY SMOMETRY -at 2 hours

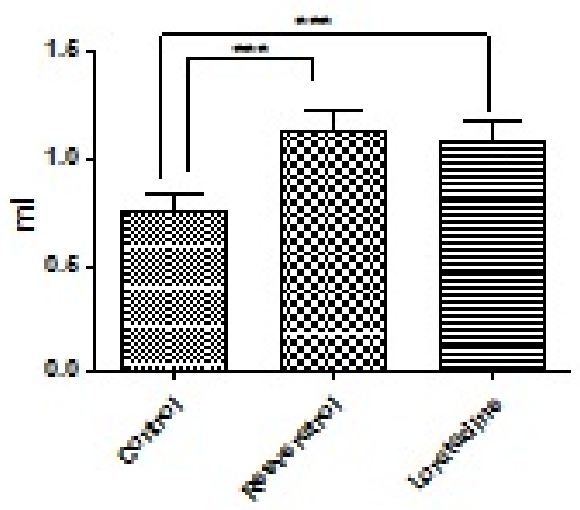

PLETHY SMOMETRY -at 24 hours

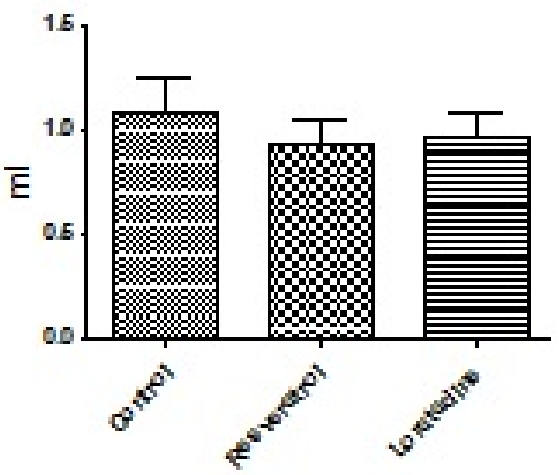

Figure 1. Plethysmometry at 2 hours and 24 hours after inflammation with carrageenan.

\section{Oxidative stress investigation}

\section{Inflamed tegument}

Loratadine significantly increased the MDA (malondialdehyde) levels in inflamed tissue, in comparison with the Control group, rats that suffered inflammation of the paw, without any other treatment $(p<0.001)$.

The concentration of glutathione (GSH) enhanced significantly in inflamed paws, in rats treated with resveratrol $(p<0.01)$ and in rats treated with loratadine $(p<0.05)$, in comparison with Control group. The group that previously received resveratrol had significant increases of the glutathione disulfide (GSSG) in comparison with the group that did not receive medication, but also in comparison with Loratadine group $(p<0.05)$. GSSG is a compound that gives information about the usage of GSH during oxidative stress reactions. The ratio between GSH (glutathione) and GSSG (glutathione disulfide) did not have significant results among the groups of rats $(p>0.05)$. 
The 2,2-diphenyl-1-picrylhydrazyl (DPPH) free radical scavenging and catalase activity did not have significant modifications among the groups $(p>0.05)$ (Figure 2).
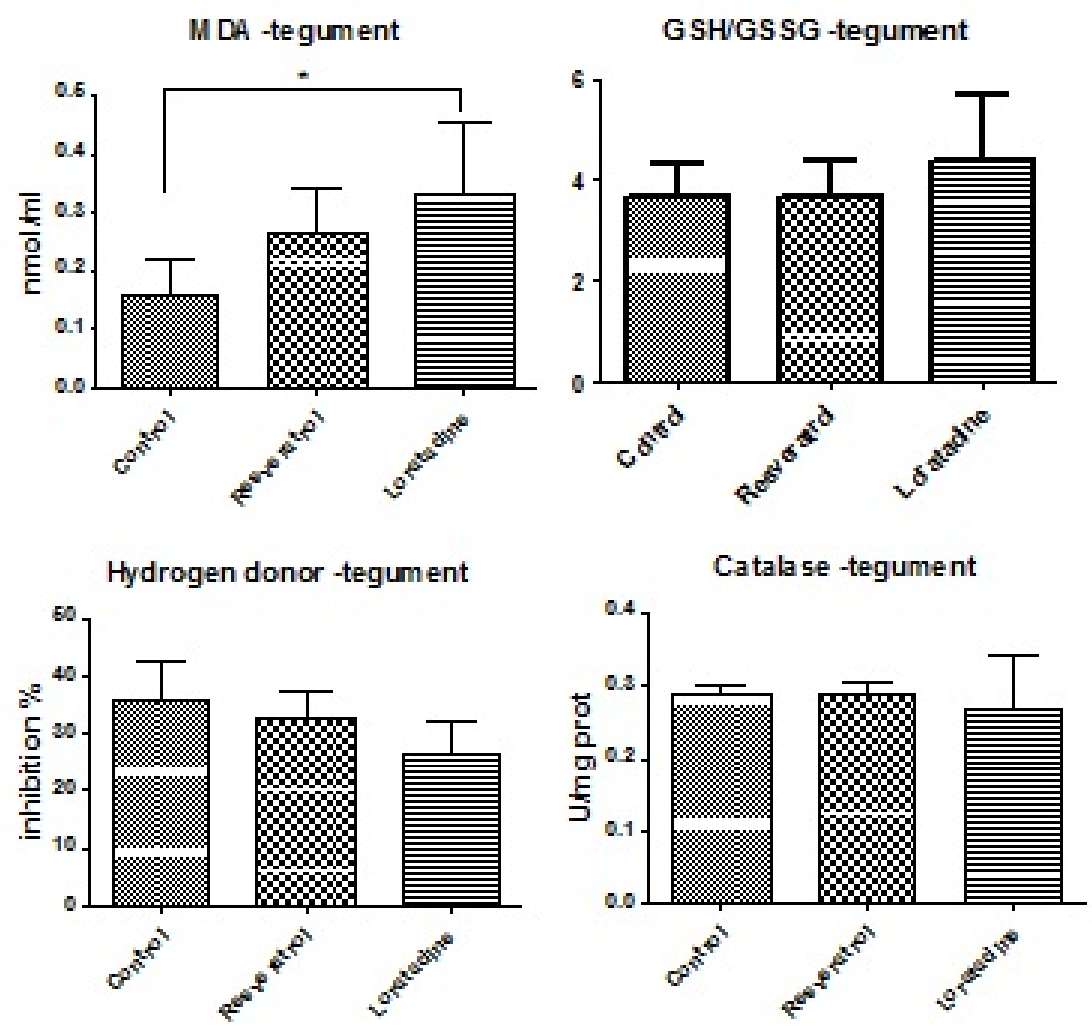

Figure 2. Malondialdehyde, ratio between glutathione and glutathione disulfide, hydrogen donor and catalase variations in inflamed tegument.

\section{Serum}

In serum, loratadine significantly increased the MDA level in comparison with resveratrol group $(p<0.05)$. The modifications between Control and Loratadine groups, and the variations between Resveratrol and Control groups were not significant ( $p>0.05)$.

The serum level of glutathione significantly increased after both treatments used (resveratrol, loratadine), in comparison with Control group $(p<0.05)$. Glutathione disulfide had no significant variation among the groups. The ratio between glutathione reduced and glutathione disulfide in serum was not influenced by the treatment with resveratrol or loratadine. 
Hydrogen donor and catalase modifications were not significant in rat's serum $(p>0.05)$ (Figure 3 ).
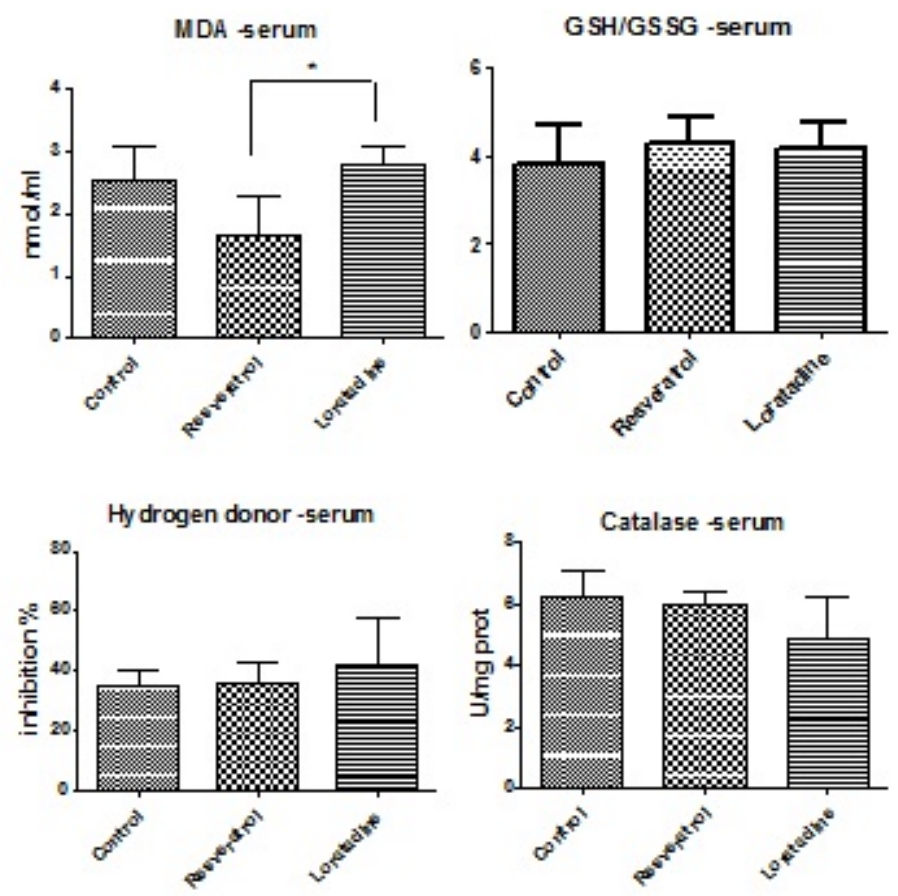

Figure 3. Malondialdehyde, ratio between glutathione and glutathione disulfide, hydrogen donor and catalase variations in serum of rats with paw tissue oedema.

\section{Liver}

Resveratrol and Loratadine groups did not present significant modifications in hepatic MDA level, in comparison with the Control group.

In liver, resveratrol and loratadine induced significantly decreases of GSH in comparison with Control group $(p<0.001)$. There were no significant modifications between the Resveratrol and Loratadine groups for GSH concentration. Glutathione disulfide concentrations in rats that received medication (Resveratrol and Loratadine groups) decreased significantly in comparison with Control group. There were no significant variations between Resveratrol and Loratadine groups for GSSG concentration. The ratio GSH/ GSSG presented a significant increase in Resveratrol group, in comparison with the Control group $(p<0.01)$ (inflammation without medication). The ratio was not significantly increased in Loratadine group.

The DPPH free radical scavenging test had not significant modifications. 
Resveratrol produced significant increases of liver catalase activity in comparison with the Control group $(p<0.05)$ and Loratadine group $(p<0.01)$ (Figure 4).

MDA -liver

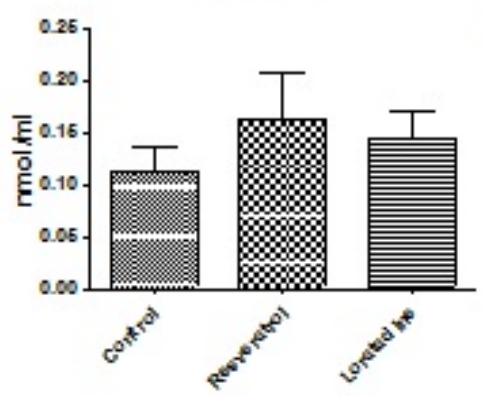

Hydrogen donor -liver

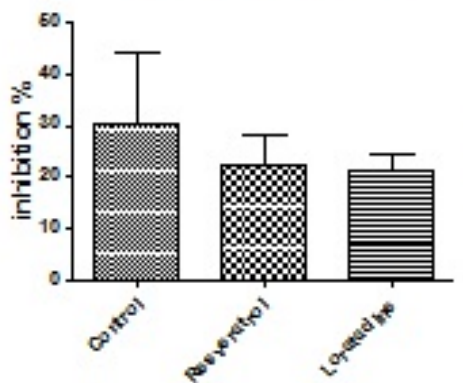

G SH/GS SG -liver
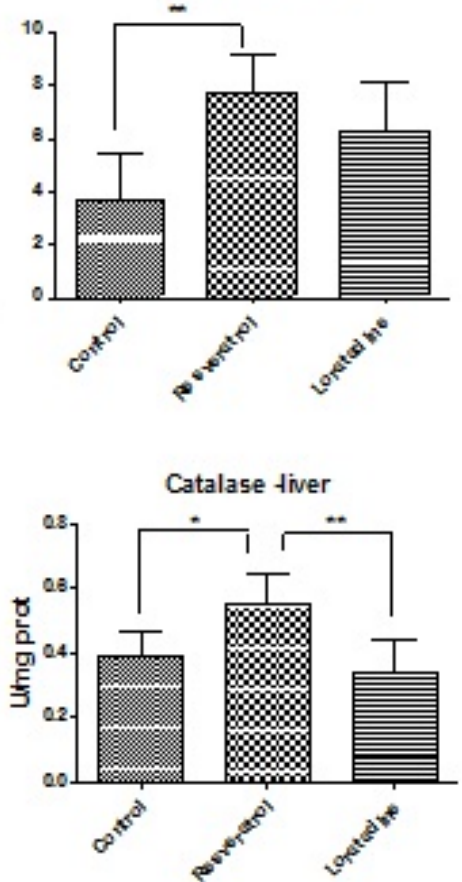

Figure 4. Malondialdehyde, ratio between glutathione and glutathione disulfide, hydrogen donor and catalase variations in the liver of rats with inflamed paw.

Histamine represents the main mediator in inflammation. Loratadine, being a selective inverse-agonist of peripheral receptor $\mathrm{H}_{1}$, could present some anti-inflammatory effects. Knowing the relationship between inflammation and oxidative stress, we decided to evaluate the loratadine effects on these processes, and at the same time, to compare its effects with those of a well-known antioxidant: resveratrol.

In our research, we investigated the oxidative stress parameters in serum, hepatic tissue and tegument tissue in rats with daily administration of resveratrol or loratadine, in experimental inflammation induced by carrageenan in rats. 
It is known that carrageenan produces free radicals that can lead to lipid peroxidation (MDA production), histamine, serotonin and bradykinin release, NO synthesis (through activation of NF-kB that leads also to $\mathrm{IL}_{s}$ and TNFa release), and tissue infiltration with neutrophils. Nitric oxide (NO) is reactive nitrogen specie that can react with many other molecules, increasing the oxidative stress; nitric oxide also produces oedema (through vasodilation) and $P G$ release [6].

Resveratrol is a polyphenol known for its antioxidant and some prooxidant effects [7]. In our study, after resveratrol administration, GSH was increased in serum and inflamed paw's tegument, without a significant variation of the ratio GSH/GSSG, proving an antioxidant potential. In the liver, both GSH and GSSG are reduced, with the significantly increase of GSH/GSSG ratio. The glutathione increase in comparison with glutathione disulfide, in our experiment, is in concordance with the study that was made by Martins et al [8] using resveratrol in activated hepatic stellate cells, that established that this polyphenol does not induce GSH oxidation. The significant decrease of GSH in the liver is realized through the GSH action against the reactive oxygen species (e.g. peroxynitrite) as it was also seen in many other studies [9][10][11]. Reduced glutathione ensures cellular protection against intracellular pro-oxidant molecules. The same results were seen in literature, with the hypothesis that resveratrol can modulate the expression of genes and proteins that are involved in tolerance to oxidative stress [12]. The catalase activity in the liver is significantly increased by resveratrol, in comparison with all other groups. This result is in concordance with studies performed in diabetic rats with liver injury [13] and in senescence-accelerated mice [14] that proved that resveratrol increases the CAT and GSH concentration in liver.

The data concerning the role of resveratrol upon inflammation are contradictory. Poulsen et al [15] and Semba et al [16] established through their researches that resveratrol does not have effects on inflammation. In addition, Gentilli et al [17] established on their model developed on rats that resveratrol administration in inflammation with carrageenan did not have effects on swelling of the paw. On the other hand, Martin et al [18] found that administration of $10 \mathrm{mg} / \mathrm{kg} /$ day of resveratrol reduced chronic inflammation developed on a colitis model. Resveratrol significantly reduces in serum IL-6 and the expression of IL-1 $\beta$ and TNFa [19].

In our study, plethysmometry performed at 2 hours after carrageenan injection shown an increase of paw's oedema under the treatment with resveratrol, indirectly emphasizing the absence of a beneficial effect upon the inflammatory process. 
Loratadine, as a selective inverse agonist of peripheral $\mathrm{H}_{1}$-receptors, binds to $\mathrm{H}_{1}$-receptors and reduces their activity [20]. Loratadine has no effect on the histamine concentration in the tissue; it does not prevent the histamine release from an inflamed tissue. Histamine is released from damaged cells and, in comparison with other tissues, its concentration in the tegument is higher.

In our experiment, loratadine significantly increased the paw's oedema measured through plethysmometry at 2 hours after carrageenan injection. Carrageenan produced free radicals that activate nuclear Factor kappa light chain enhancer of activated B cells (NF-kB) that regulates inflammation through nitric oxide synthesis (NO can promote prostaglandin release, vasodilation, oedema, new reactive species synthesis), cyclooxygenase activation, the release of $\mathrm{TNF} \alpha, \mathrm{IL}-1 \beta$. Desloratadine, the metabolite of loratadine can inhibit the activity of $\mathrm{H}_{1}$-receptors, blocking the NF-kB activation, leading to an inhibition of cytokines release [21]. Histamine, serotonin, and bradykinin are the first measurable mediators produced in carrageenan inflammation. Later, prostaglandin, lysosomal enzymes and protease are released. The significant increase of paw inflammation in rats that received loratadine cannot be explained by these mechanisms presented in literature. However, carrageenan, through free radicals, promotes lipid peroxidation that can lead to oedema, and it directly causes neutrophil infiltration that can produce new oxygen species (like superoxide) that continue the inflammation processes. Cheng et al [22] established on an experimental model performed on A549 cells that loratadine has anti-inflammatory effects through inhibition of IL-8. Amsellen et al [23] evaluated the loratadine effects on PMN cocultured with HAEC and assessed that anti-inflammatory effect is realized through reduction of LTB4 synthesis by neutrophils. From the best of our knowledge, loratadine was not used in a study on inflammation with carrageenan in rats, and in our study, we established that the administration of this selective inverse agonist of peripheral $\mathrm{H}_{1}$-receptors increases the paw's oedema. These differences between our experiment and other studies can be explained through the following effects. Loratadine increases the cytosolic calcium by releasing it from intracellular stores (calcium activates NF-kB, increases the cytokines release), partially inhibits the leukotrienes as Letari et al [24] assessed in their research performed in vitro on rat peritoneal macrophages. Naclerio [25] in a study performed on nasal challenge antigen model and Shroeder et al [26] in their research in vitro on human basophils established that loratadine reduces the release of histamine and $\mathrm{PGD}_{2}$, but not significantly. The anti-inflammatory effects of loratadine are not efficient in reducing the inflammation, and it promotes a supplementary oxidative stress in the inflamed tissue. Loratadine inhibits $\mathrm{H}_{1}$-receptors but does not inhibit the 
histamine release. Loratadine and its metabolite desloratadine do not inhibit the release of $\mathrm{PGD}_{2}$, cytokines, LTC4 -factors that promote inflammation. In our study, loratadine administration significantly increased MDA concentration in comparison with the rats that suffered inflammation without treatment. This lipid peroxidation promoted the oedema that was found significantly increased through plethysmometry.

Regarding the effects of loratadine upon oxidative stress in our study, loratadine increased the GSH level in serum. These results are similarly to those found by Sadowska-Woda [27] in a study performed in vitro on human erythrocytes.

In liver, loratadine significantly decreased GSH and GSSG levels, without the modification of the GSH/GSSG ratio. These results indirectly prove the occurrence of oxidative stress, but the reactive species that were formed, were scavenged by GSH. Loratadine is processed in liver, and Yumibe et al [28] established through their experiment performed on human liver microsomes that at the first passage through this organ, loratadine is almost complete metabolized by cytochrome P450 to several substances, and one of these is desloratadine. CYP3A4 and CYPP2D6 are the enzymes of the cytochrome P450 that mediate the metabolism of loratadine till the important active metabolite: decarboethoxyloratadine [29]. In literature, loratadine and its metabolites are presented like medications that can affect rarely the liver structures, and they can cause liver modifications when they are administrated with substances that can interfere with the cytochrome P450 enzymes. Our study assessed the presence of an oxidative stress that is neutralized by the scavenger activity made by GSH.

The performed experimental study is useful because our results complete the other researches data.

For the clinicians, the obtained data can help them in choosing the treatment. Anti-allergic treatment in persons that also develop an inflammation should be done with another anti-histaminic (loratadine administration gave discouraging results).

Further studies should be performed to identify more markers of inflammation that are stimulated or inhibited by resveratrol and loratadine.

\section{CONCLUSIONS}

In inflamed skin, resveratrol did not ensure protection, increasing the inflammation, and loratadine developed a supplementary oxidative stress increasing lipid peroxidation and inflammation. 
In serum, resveratrol and loratadine had antioxidant effects, stimulating the production of GSH.

In liver, resveratrol had antioxidant activity, while loratadine developed a minor oxidative stress.

\section{EXPERIMENTAL SECTION}

\section{Methods}

24 adult male rats, Wistar breed, with weight $200-220 \mathrm{~g}$, were randomly allocated into 3 groups, with eight rats in each group.

The cages were kept in the same room, in the same environmental standard conditions at $22+/-1^{\circ} \mathrm{C}$, a daylight cycle of 12 hours, having, before the study, one week for adaptation to these conditions. The substances were administered by oral gavage, between 7 a.m. - 8 a.m. to all the rats. The experiments were done in the biobase of U.M.Ph. "Iuliu Haţieganu" Cluj-Napoca. All the procedures were performed with the approval of the Ethical Committee of U.M.Ph. "Iuliu Haţieganu" Cluj-Napoca and respected the Directive 86/609/EEC.

The groups were the following:

Control group -received physiological salt, $0.5 \mathrm{ml} /$ day by oral gavage, for 14 days, at the same hour when the test groups received medication

Resveratrol group - for 14 days, received $50 \mathrm{mg} / \mathrm{kg} /$ day of resveratrol, through oral gavage (resveratrol gelatinous capsule, each capsule containing 100 mg dry extract 200:1 from Polygonum cuspidatum root, standard resveratrol $50 \%$, Polypharma Industries S.R.L.)

Loratadine group - received $0.5 \mathrm{ml}$ of solution containing $0.14 \mathrm{mg} / \mathrm{kg} / \mathrm{day}$ of loratadine (Claritin) in physiological salt, by oral gavage, for 14 days. (Claritin: $10 \mathrm{mg}$ of loratadine, Schering-Plough Labo N.V. Belgium).

On the $14^{\text {th }}$ day of the treatment, after 30 minutes from the medication administration, all the rats were intraplantar injected with $0.2 \mathrm{ml}$ of carrageenan $1 \%$ (fresh prepared solution of $1 \%$ carrageenan in saline, Sigma-Aldrich Co. LLC, Germany), in the posterior right paw, to produce them a paw inflammation. The inflammation of the rat's paw was measured through plethysmometry using a (plethysmometer UGO BASILE North America, 7140). For all the rats, measures of the injected paw were done at 2 hours and 24 hours after the carrageenan administration. After the plethysmometry was done, the obtained values were used in the following formula:

\section{Anti-inflammatory activity (\%) $=[1$-treated paw volume/untreated paw volume] 100}


In the day 15, the blood samples were taken and under anaesthesia with ketamine and xylazine $(90 \mathrm{mg} / \mathrm{kg}$ b.w. ketamine and $10 \mathrm{mg} / \mathrm{kg}$ b.w. xylazine) paw's skin and liver were collected.

The oxidative stress was investigated in serum, inflamed paw's tissue and liver through the following parameters: malondialdehyde (MDA), glutathione (GSH), oxidised glutathione (GSSG), ratio GSH/GSSG, DPPH free radical scavenging method and catalase activity.

Malondialdehyde (MDA), product of lipid peroxidation was determined using the method of Conti [30]. Reduced glutathione (GSH) was determined using the method described by Hu [31] and oxidized glutathione (GSSG) using Vats' method [32]. The ratio GSH/GSSG was calculated, because it is an indicator of the oxidative stress. For DPPH test, the method of Janaszewska was used [33]. For evaluation of catalase activity the method described by Pippenger et al [34] was used.

The obtained data were statistically processed using GraphPad Prism version 5.03 for Windows, GraphPad Software, (San Diego California USA), performing One-way ANOVA followed by the Post-test Bonfferoni. It was set the threshold significance level at $p<0.05$.

\section{REFERENCES}

1. M. Matsubara, Biochem Pharmacol, 2005, 69, 433.

2. B. Tian, A.R. Brasier, Recent Prog Horm Res, 2003, 58, 95.

3. A.R. Brasier, Cardiovascular Toxicology, 2006, 6(2), 111.

4. S. Sas, D.K. Das, Inflamm Allergy Drug Targets, 2007, 6 (3), 168.

5. R.L.Wu, J.C. Anthes, W. Kreutner, A.G. Harris, R.E. Jr. West, Int Arch Allergy Immunol, 2004, 135 (4), 313.

6. F.R.F. Silva, C.M.P.G. Dore, C.T. Marques et al, Carbohydrate Polymers, 2010, $79,26$.

7. C.A. De la Lastra, I. Villegas, Biochemical Society Transactions, 2007, 35 (Pt 5), 1156.

8. L.A. Martins, B.P. Coelho, G. Behr, et al., Cell Biochem Biophys, 2014, 68, 247.

9. C. Harris, J.M. Hansen, Methods in Molecular Biology, 2012, 889, 325.

10. L. Yuan, N. Kaplowitz, Molecular Aspects of Medicine, 2009, 30(1-2), 29.

11. H.J. Forman, H. Zhang, A. Rinna, Molecular Aspects of Medicine, 2009, 30 (1-2), 1.

12. M. Ruweler, M. Gulden, E. Maser, M. Murias, H. Seibert, Chemico-Biological Interactions, 2009,182 (2-3), 128.

13. N. Hamadi, A. Mansour, M.H. Hassan, F. Khalifi-Touhami, O. Badary, J Biochem Molecular Toxicology, 2012, 26(10), 384. 
D. MITREA, S. CLICHICI, A. FILIP, D. OLTEANU, I. BÂLDEA, R. MOLDOVAN, N. DECEA, O. HOTEIUC

14. G. Liu, Z. Zhang, B. Yang, W. He, Journal of Medicinal Plants Research, 2012, $6(2), 325$.

15. M.M. Poulsen, P.F. Vestergaard, B.F. Clasen, et al., Diabetes, 2013, 62, 1186.

16. R.D. Semba, L. Ferrucci, B. Bartali, et al., JAMA Intern Med, 2014, 174(7), 1077.

17. M. Gentilli, J.X. Mazoit, H. Bouaziz, et al., Life Sci, 2001, 68(11), 1317-21.

18. A.R. Martin, I. Villegas, M. Sánchez-Hidalgo, C. Alarcón de la Lastra, Br J Pharmacol, 2006, 147(8), 873.

19. J. Tomé-Carneiro, M. Larossa M, M.J. Yáňez-Gascón, et al., Pharmacol Res, 2013, 72, 69.

20. R. Leurs, M.K. Church, M. Tagliatela, Clinical and Experimental Allergy, 2002, 32(4), 489.

21. G.W. Canonica, M. Blaiss, World Allergy Organ J, 2011, 4(2), 47-53.

22. K.C. Cheng, J.Y. Hsu, L.S. Fu, W.C. Chang, J.J. Chu, C.S. Chi, J Microbiol Immunol Infect, 2006, 39, 206.

23. C. Amsellen, W. Czarlewki, M. Lagarde, Y. Pachéco, Pulmonary Pharmacology \& Therapeutics, 1998, 11(4), 245.

24. O. Letari, A. Miozzo, G. Folco, P.A. Belloni, A. Sala, G.E. Rovati, S. Nicosia, Eur $J$ Pharmacol, 1994, 15, 266(3), 219.

25. R.M. Naclerio, Ann Allergy, 1993, 71(3), 292.

26. J.T. Schroeder JT, R.P. Schleimer, L.M. Lichtenstein, W. Keutner, Clin Exp Allergy, 2001, 31(9), 1369.

27. I. Sadowska-Woda, B. Sychta, M. Rachel, E. Bieszczad-Bedrjczuk, Environmental Toxicology and Pharmacology, 2010, 30(2), 141.

28. N. Yumibe, K. Huie, K.J. Chen, M. Snow, R.P. Clement, M.N. Cayen, Biochem Pharmacol, 1996, 51, 165.

29. B. Katchen, J. Crame, M. Chung, Ann Allergy, 1985, 55, 393.

30. M. Conti, P.C. Morand, P. Levillain, A. Lemonnier, Clinical Chemistry, 1991, 37, 1273.

31. M.L. Hu, Methods in Enzymology, 1994, 233, 380.

32. P. Vats, V.K. Singh, S.S. Sing, Aviation, Space and Environmental Medicine, 2008, 79 (12), 1106.

33. A. Janaszewska, G Bartosz, Scandinavian Journal of Clinical and Laboratory Investigation, 2002, 62 (3), 231.

34. C.E. Pippenger, R. W. Browne, D. Armstrong, Methods in Molecular Biology, 1998, 108, 299. 